\title{
Concurrent Development of the Head and Pinnae and the Acoustical Cues to Sound Location in a Precocious Species, the Chinchilla (Chinchilla lanigera)
}

\author{
Heath G. Jones ${ }^{1,2}$, Kanthaiah Koka ${ }^{2}$, Jennifer L. Thornton ${ }^{1,2}$, and Daniel J. Tollin ${ }^{1,2,3}$ \\ ${ }^{1}$ Neuroscience Training Program, University of Colorado Denver School of Medicine, Aurora, CO 80045, USA \\ ${ }^{2}$ Department of Physiology and Biophysics, University of Colorado Denver School of Medicine, Mail Stop 8307 PO Box 6511 \\ 12800, East 19th Avenue, Aurora, CO 80045, USA \\ ${ }^{3}$ Department of Otolaryngology, University of Colorado Denver School of Medicine, Aurora, CO 80045, USA
}

Received: 28 July 2010; Accepted: 1 October 2010; Online publication: 19 October 2010

\begin{abstract}
Sounds are filtered in a spatial- and frequency-dependent manner by the head and pinna giving rise to the acoustical cues to sound source location. These spectral and temporal transformations are dependent on the physical dimensions of the head and pinna. Therefore, the magnitudes of binaural sound location cues - the interaural time (ITD) and level (ILD) differences-are hypothesized to systematically increase while the lower frequency limit of substantial ILD production is expected to decrease due to the increase in head and pinna size during development. The frequency ranges of the monaural spectral notch cues to source elevation are also expected to decrease. This hypothesis was tested here by measuring directional transfer functions (DTFs), the directional components of head-related transfer functions, and the linear dimensions of the head and pinnae for chinchillas from birth through adulthood. Dimensions of the head and pinna increased by factors of 1.8 and 2.42, respectively, reaching adult values by $\sim 6$ weeks. From the DTFs, the ITDs, ILDs, and spectral shape cues were computed. Maximum ITDs increased by a factor of 1.75 , from $\sim 160 \mu$ s at birth (P0-1, first postnatal day) to $280 \mu$ s in adults. ILDs depended on source location and frequency exhibiting a shift in the frequency range of substantial ILD
\end{abstract}

Correspondence to: Daniel J. Tollin - Department of Physiology and Biophysics - University of Colorado Denver School of Medicine · Mail Stop 8307 PO Box 6511 12800, East 19th Avenue, Aurora, CO 80045, USA. Telephone: +1-303-7240625; fax: +1-303-7244501; email: Daniel.tollin@ucdenver.edu
$(>10 \mathrm{~dB})$ from higher to lower frequencies with increasing head and pinnae size. Similar trends were observed for the spectral notch frequencies which ranged from $14.7-33.4 \mathrm{kHz}$ at $\mathrm{P} 0-1$ to $5.3-19.1 \mathrm{kHz}$ in adults. The development of the spectral notch cues, the spatial- and frequency-dependent distributions of DTF amplitude gain, acoustic directionality, maximum gain, and the acoustic axis were systematically related to the dimensions of the head and pinnae. The dimension of the head and pinnae in the chinchilla as well as the acoustical properties associated with them are mature by $\sim 6$ weeks.

Keywords: interaural time difference, interaural level difference, spectral shape cues, sound localization, development

\section{INTRODUCTION}

Sound source location is based on three acoustical cues. Interaural level differences (ILDs) are the differences in the sound pressure levels at each ear that result jointly from amplification of sound at the ipsilateral ear and attenuation at the contralateral ear from the "acoustical shadow" cast by the head. Interaural time difference (ITD) cues arise from the fact that the two ears are separated in space by the head, so a sound traveling from a source to one side of the animal arrives at the near ear a short time before the far ear. Finally, for sounds positioned along 
the vertical plane, the direction-dependent acoustic filtering properties of the torso, head and the pinna give rise to "spectral shape" cues (Tollin and Yin 2003).

The magnitudes of the cues to location are a complex function of both frequency and location, but also critically depend on the physical size of the head and pinnae. These facts create a challenge during early auditory development where head and pinnae size increase dramatically in humans and animals, thus changing the relationship between the cues and location (Tollin and Koka 2009a, b). An attractive hypothesis is that auditory experience during development calibrates the neural circuits that process sound location to the mature acoustical properties of the head and ears of each individual (Moore and King 2004). As a first step in testing this hypothesis, it is necessary to measure the developmental changes in the magnitudes of the acoustical cues to location and the time course over which they occur.

Here, we describe the development of the head and pinnae in the chinchilla (Chinchilla lanigera) along with the concommittant development of the cues to location. The chinchilla has been an important model for auditory neuroscience for several reasons. The chinchilla exhibits an audiogram similar to humans (the average hearing range of the chinchilla extends from $50 \mathrm{~Hz}$ to $33 \mathrm{kHz}$ ) and their auditory system exhibits similar physiological, anatomical, and behavioral characteristics as those of humans and higher primates (Miller 1970; Heffner and Heffner 1991; Niemiec et al. 1992). Additionally, chinchillas have good sound location acuity for sources varying in both azimuth (Heffner et al. 1994) and elevation (Heffner et al. 1995) and the anatomy and physiology of the binaural auditory system is comparable (Chen and Sinex 1999; Nuding et al. 1999; Alkhatib et al. 2006) to other mammalian animal model systems for binaural hearing. Yet unlike many common mammalian animal models (e.g., mouse, rat, cat, ferret, and gerbil), which are born with immature and non-functional auditory systems, the chinchilla is a precocious species, born with a nearly fully functioning peripheral auditory system (Harrison et al. 1996; Heffner et al. 1996). In this regard, the chinchilla is similar to the human and as such may provide a more appropriate animal model to examine the role of experience in the development of the binaural auditory system.

\section{METHODS}

\section{Animal subjects and preparation}

All surgical and experimental procedures complied with the guidelines of the University of Colorado-
Anschutz Medical Campus Animal Care and Use Committees and the National Institute of Health. Nineteen chinchillas (eight adults, 11 infants) of various ages were used for acoustical measurements in this study. Adult animals were obtained from a vendor (Moulton Chinchilla Ranch, MN), and infants were produced onsite in our animal care facility. Linear measurements of the head and pinna were performed on all of these 19 animals, plus an additional 55 adult animals that were used for other anatomical and physiological experiments. The latter measurements were used for generating distributions of adult head and pinnae measurements.

Animals used for acoustical measurements were anesthetized with an initial intramuscular (IM) dose of ketamine hydrochloride (KetaVed, $30 \mathrm{mg} / \mathrm{kg} \mathrm{IM}$ ) and xylazine hydrochloride (TranquiVed, $5 \mathrm{mg} / \mathrm{kg}$ IM). Supplementary injections of ketamine $(15 \mathrm{mg} /$ $\mathrm{kg} \mathrm{IM})$ and xylazine $(2.5 \mathrm{mg} / \mathrm{kg}$ IM) were administered to maintain an adequate level of anesthesia every $\sim 1 \mathrm{~h}$ based on reflex response to a paw pinch and changes in physiological parameters (heart rate, etc.). Heart rate, blood-oxygen levels $\left(\mathrm{Sp}_{2}\right)$, respiratory rate, and end-tidal $\mathrm{CO}_{2}$ were monitored continuously via a capnograph (Surgivet V90040, Waukesha, $\mathrm{WI})$ and displayed graphically in real-time on a PC monitor. Body temperature was continuously monitored with a rectal probe and maintained with a heating pad at $37^{\circ} \mathrm{C}$. Following the initial dose of anesthesia, a tracheal cannula was inserted and secured with nylon string. The animal was placed into a custom head holder which held the head securely but that did not interfere with the direct propagation of sound waves from the loudspeakers to the external ear. A small $(\sim 2-3 \mathrm{~mm})$ incision was made to expose the lateral aspect of the bony external auditory canal. Silicone probe tubes, $50 \mathrm{~mm}$ in length (Bruel and Kjaer, part no. AF-0555, $1.65 \mathrm{~mm}$ outer diameter) connected to microphones (Bruel and Kjaer, type 4182) were positioned within $<2 \mathrm{~mm}$ of the tympanic membrane by drilling a small hole through the bone $3 \mathrm{~mm}$ proximal to the beginning of the bony portion of the external auditory canal. The probe tubes were then advanced under otoscopic visualization towards the tympanic membrane and fixed into place with cyanoacrolate adhesive. This procedure does not alter the pinnae in any way. The pinnae themselves were supported from behind by thin wire in an erect position similar to the pinnae position the chinchillas adopt when awake and alert.

\section{Experimental setup and stimuli presentation}

For acoustic measurements, experiments were performed in a double-walled, sound-attenuating chamber (Industrial Acoustics Company, Bronx, NY). 
Stimuli were generated in MATLAB ${ }^{\circledR}$ (v7.1, The Math Works Inc., Natick, MA) and presented using the digitalto-analog converters of a Tucker Davis Technologies (TDT) RX6. Microphone responses at both ears were simultaneously recorded. All stimulus presentation, acquisition, and processing was done using custom software in MATLAB ${ }^{\circledR}$.

Stimuli were presented from 25 response-matched speakers (Morel MDT-20) attached to a semicircular boom and placed every $7.5^{\circ}$ from $-90^{\circ}$ (left) to $+90^{\circ}$ (right). The axis of rotation of the boom was aligned with the interaural axis of the animal (i.e., through the ears). The radius of the boom was $1 \mathrm{~m}$. A stepper motor (Advanced Mirco Systems AMH34-1303-3) and motor controller/supply (Advanced Micro systems CMAX-810) under computer control could position the boom in elevation with a precision of $<1^{\circ}$. The semicircular boom was moved in steps of $7.5^{\circ}$ along the elevations using the stepper motor controlled via personal computer by custom-written software in MATLAB $^{\circledR}$. Stimuli were presented from a total of 325 locations selected to evenly sample azimuth and elevation (i.e., the pole locations were not overly sampled). The elevations spanned from $-45^{\circ}$ (low elevations in front of the animal) to $+90^{\circ}$ (directly above the animal). Additional measurements were made for a few animals for 525 locations spanning from elevations of $-45^{\circ}$ (low elevations in front of the animal) to $+180^{\circ}$ (directly behind the animal).

The acoustical measurement stimuli consisted of 11th order maximum length sequences (MLS) (Rife and Vanderkooy 1989) repeated without interruption 128 times from each loudspeaker. The MLS were presented at full 24-bit resolution with a sampling rate of $97,656.25 \mathrm{~Hz}$. A single sequence of the 11th order MLS is comprised of 2,047 $\left(2^{11}-1\right)$ samples and is $20.96 \mathrm{~ms}$ in duration. The resulting acoustic waveforms in the ear canals of the left and right ears were simultaneously recorded through probe tubes that were connected to the microphones, amplified (TDT MA2) and collected at 24 bits using two analog to digital converter channels at $97,656.25 \mathrm{~Hz}$ (TDT RX6). The recorded signals were stored on a PC hard disk for later processing. A calibration measurement was also made in the absence of the animal by placing the tips of the probe tubes so that they corresponded to the location where the center of the chinchilla's head would be located. The calibration measurements captured the spectral characteristics of the loudspeakers and microphones.

\section{Data processing and data analysis}

The free-field-to-eardrum impulse response for each ear and each location was calculated as previously described (Tollin and Koka 2009b) by circular cross- correlation of the original 11th order MLS stimulus and the in-ear recording from the probe tube microphones (Rife and Vanderkooy 1989). The impulse responses for each ear were truncated to 512 points (5.12-ms duration) by a 512-point Hanning window where the center of the window was set to coincide with the point of maximum amplitude in the impulse response. The windowing process removes the smallamplitude reflections that may be contained in the impulse response. Moreover, these raw impulse responses contained not only the desired spectral and temporal transformations of sound by the head and pinna, but also the loudspeaker, microphone, and measurement-system frequency responses. These equipment-related frequency responses were removed by dividing the Fourier transforms of each of the impulse responses by that of the appropriate calibration measurements made for each loudspeaker by placing the microphone tips to where the animal's head would be. The resulting functions are referred to as the head-related transfer functions (HRTFs), representing the acoustical gain and delay introduced by the head and pinna. From the HRTFs, the directional transfer functions (DTFs) were calculated for each ear by dividing the HRTF made at each spatial location by the geometrical mean of all the measured HRTFs across all measurement locations for that ear (Middlebrooks and Green 1990). In essence, the DTFs are the sound source direction-dependent components of the HRTFs.

The amplitude spectra of the DTFs were calculated after the spectra were passed through a bank of 350 band pass filters, the center frequencies of which were spaced at intervals of 0.0143 octave spanning from 0.25 to $32 \mathrm{kHz}$. The 3-dB bandwidth of filters was held constant across all frequencies at 0.0571 octaves, and the upper and lower slopes of the filters fell off at $\sim 105 \mathrm{~dB}$ /octave. These filters have properties similar to the bank of triangular band pass filters described in the literature (Xu and Middlebrooks 2000; Schnupp et al. 2003; Koka et al. 2008).

Several acoustical features of the DTFs were examined. Some features include the broadband spectral "notches," which are prominent energy minima in the DTFs. Other features could be computed directly from the DTFs, such as the spatial and frequency dependence of the acoustical gain and the "acoustic axis," which is the spatial location of the maximum gain for a given frequency (Middlebrooks and Pettigrew 1981). The two binaural cues to sound location were also computed. The ILD spectrum was derived by computing the differences (in dBs) in the DTFs, frequency by frequency between right and left ears at all elevations and for all azimuthal angles. A positive ILD indicates that the decibel level at the right ear was higher than the decibel level at the left 
ear. The ILDs for particular frequencies and locations were extracted from the ILD spectra. The lowfrequency ongoing ITDs in the fine structure of the sounds were measured by computing for each spatial location the cross-correlation of the raw head-related impulse responses (i.e., before conversion to DTFs) of the left and right ears after low-pass filtering (5th order Butterworth) with a $3.5 \mathrm{kHz}$ cut off and by finding the delay corresponding to the maximum point in the cross-correlation function. This process was repeated for each location.

Although the audiogram of chinchilla ranges up to $\sim 32 \mathrm{kHz}$ (Heffner and Heffner 1991), the data in the current manuscript are considered only up to $30 \mathrm{kHz}$. To determine whether the probe tube microphone and loudspeaker combination was sufficient for these measurements, we used the technique described by Rice et al. (1992). Here, the frequency response from the center loudspeaker $\left(0^{\circ}, 0^{\circ}\right)$ was measured as described in the calibration measurement methods above. Next, the tip of the probe tube was completely blocked with a silicone impression material (Silicone, Westone, Colorado Springs, CO, USA) and the frequency response remeasured. By comparing these two frequency responses, we found that the microphone, probe tube and loudspeaker combination was sufficient to measure signal attenuations (i.e., those that would be due to the head and/or pinnae) of at least $35 \mathrm{~dB}$ at $30 \mathrm{kHz}$, and could measure attenuations of $40 \mathrm{~dB}$ or more for lower frequencies.

For spatial plotting purposes, the data were displayed as Aitov projections (Bugayevskiy and Snyder 1995). In this projection, the nose of the animal is considered to be projecting out of the page at $0^{\circ}$ azimuth and $0^{\circ}$ elevation, as if the animal were looking at the reader. The spatial plots were plotted for frontal data for elevations from $-45^{\circ}$ to $+90^{\circ}$ and azimuth from $+90^{\circ}$ to $-90^{\circ}$. Unweighted linear regressions were performed using the curve fitting package in SigmaPlot (V10, Systat Software, Inc., Richmond, CA).

\section{RESULTS}

The development of the linear dimensions of the head and pinna reported here were based on 25 chinchillas which were measured approximately every day starting within $24 \mathrm{~h}$ of birth, or postnatal $(\mathrm{P})$ day P0-1. Not all infants were measured on all days or through adulthood, as some animals were used for acoustical measurements before reaching maturity. To establish adult distributions, measurements were made of the head, pinnae and weight in 55 adults prior to other ongoing physiological studies in the laboratory. For acoustical measurements, 19 chinchil- las were used; eight were adults and 11 were at ages ranging from $\mathrm{P} 0$ to $\mathrm{P} 42$ (particular focus was given to ages $\mathrm{P} 0-1, \mathrm{P} 7, \mathrm{P} 14, \mathrm{P} 28$, and $\mathrm{P} 42$ ).

\section{Developmental growth of head and pinna dimensions}

Head diameter was taken at the widest part of the skull, occurring a few millimeters rostral to the meatus at the points of the zygomatic process (the bizygomatic breadth) of the temporal bone (Gilbert 1981); this is the maximum transverse diameter of the head. Following Coles and Guppy (1986), in order to better account for the contributions of both the height and width dimensions of the oval-like-shaped pinna (Fig. 1B, inset) to the
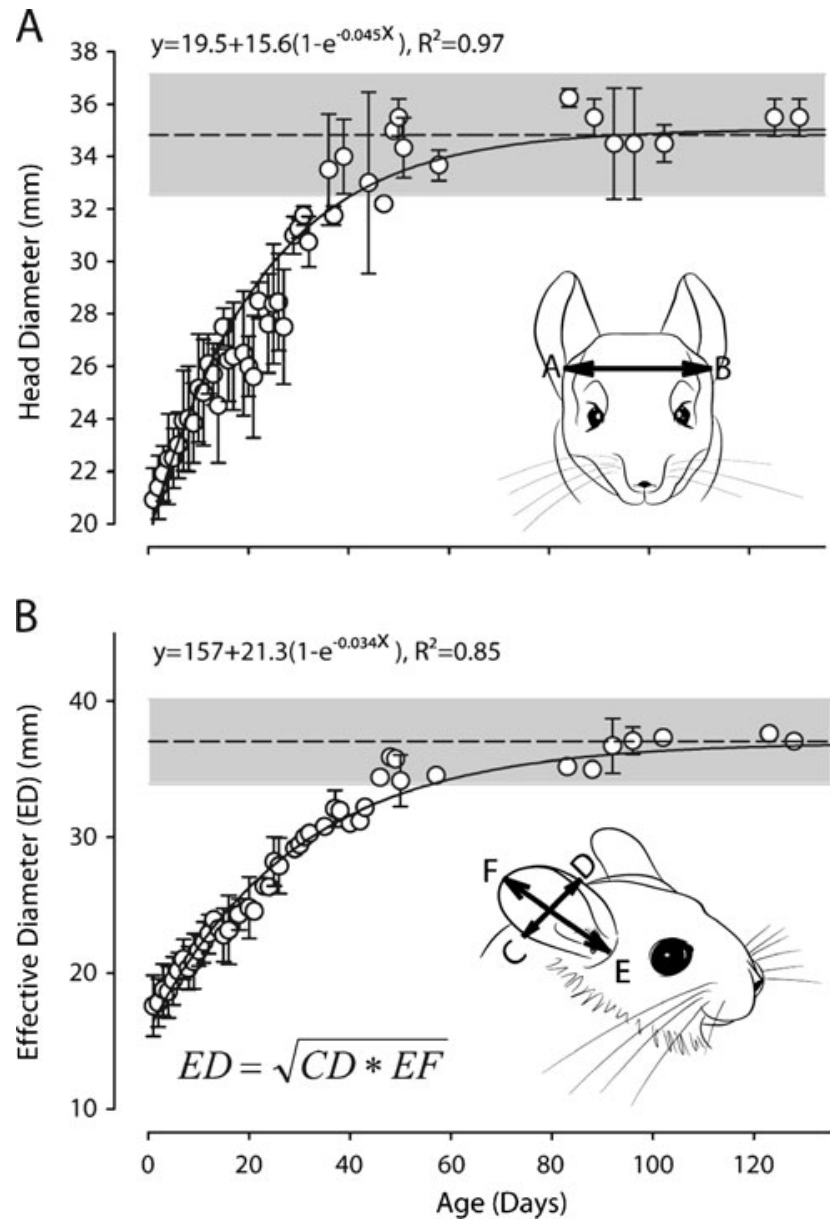

FIG. 1. Developmental growth of the dimensions of the head and pinnae of the chinchilla. The measured dimensions are shown in the insets of $(\mathbf{A})$ and $(\mathbf{B})$. A Head diameter $(A B)$. B Pinna width at halfheight $(C D)$ and pinna height $(E F)$. Here, the effective diameter $(E D$, equation in inset of (B)) for the pinnae were reported. The measured data are from 19 animals. Data points with error bars indicated across-animal mean $\pm S D$ of the measured dimension at that age. Data points without error bars indicate single animal measurements at that age. In both panels, the dashed line and the gray area indicates the mean and associated SD of the dimensions, respectively, measured in 55 adult animals. 
transformations of sounds, we computed the effective diameter of the pinnae. The effective diameter, given by the square root of the product of pinna height and pinna width (see equation, inset of Fig. 1B), is based on an equal areas approximation to a circular aperture. (The elipse equal-area approximation to a circle is derived by setting the area of an elipse given by $\pi^{*} A^{*} B$ where $A$ and $B$ are the radii of the long and short axes of the elipse to that of a circle whose area is given by $\pi^{*} R^{2}$ where $R$ is the radius. Solving this equality for $R$ yields $\operatorname{sqrt}\left(A^{*} B\right)$.) This approximation allows the use of a circular aperture model (derived from Calford and Pettigrew (1984), and Coles and Guppy (1986)) to predict the frequency dependence of sound wave interaction with an oval shaped structure like the chinchilla pinnae.

The head, pinnae and weight measurements were made in 55 adults (mean weight, $567 \pm 110 \mathrm{~g}$; head diameter $=35.5 \pm 1.8 \mathrm{~mm}$; pinna height $=49.8 \pm 3.5 \mathrm{~mm}$; pinna width $=28.14 \pm 3.2 \mathrm{~mm} ; n=55$ ). These distributions were used to establish the mean adult dimension for each measurement and corresponding standard deviations (gray bars, Fig. 1A, B) to which the developing dimensions could be compared. In 11 animals, at $\mathrm{P} 0-1$, the mean weight $(44.7 \pm 7 \mathrm{~g})$, head size $(19.8 \pm 1.8 \mathrm{~mm})$, pinna height $(17.3 \pm 1.7)$, and pinna width $(12.8 \pm 2.8 \mathrm{~mm})$ were considerably smaller than adult values (above). Figure 1 shows linear measurements of head diameter and effective pinna diameter as a function of age in days for 25 chinchillas starting at P0. Individual symbols represent across animal mean values if an error bar (standard deviation) is present; otherwise, the symbol represents the measurement of a single animal at that day. The horizontal lines in each panel represent the mean for these values in the 55 adults and the shaded gray area represents the associated standard deviation. Based on fitted growth curves (Fig. 1), head diameter increased by a factor of 1.8 from $19.8 \mathrm{~mm}$ at $\mathrm{P} 0-1$ to $35.5 \mathrm{~mm}$ in adults and first reached the adult range by $\mathrm{P} 42$ or 6 weeks. The pinnae height and width grew at slightly different rates. Pinnae height increased by a factor of 2.89 from 17.3 to $49.8 \mathrm{~mm}$, reaching the adult range by 6 weeks and pinnae width increased by a factor of 2.2 from 12.8 to $28.14 \mathrm{~mm}$, reaching adult range between 5 and 6 weeks (not shown). The effective pinnae diameter (see Fig. 1B) increased by a factor of 2.42 from 15.32 to $37.03 \mathrm{~mm}$, reaching adult range by $\sim \mathrm{P} 45$.

To quantify the growth rate, a three-parameter exponential rise to maximum function was fitted to the data of the form $y=y_{0}+a\left(1-e^{-\mathrm{bx}}\right)$, where $x$ is the age in days, $y_{0}$ is the dimensions at birth (P0), $a$ is the amount by which that dimension increases during development, $\left(y_{0}+a\right)$ is the asymptotic value at full development, and $b$ is the rate of growth. This equation accurately characterized the growth of each dimension (based on $F$ test, $p<0.0001$ for all fitted equations; coefficients of determination of the fit, $R^{2}$, are reported in each figure). The fitted parameters of the equations are shown in each panel in Figure 1.

Here we define maturation of the head and pinnae dimensions as the postnatal day at which the measured dimensions first fell within the adult range of values (shaded bar, Fig. 1) and remained in that range at subsequently older ages. Based on this definition as well as the fitted growth curves, head diameter at $\mathrm{P} 0-1$ was on average $46 \%$ smaller than adult, almost doubling from $19 \mathrm{~mm}$ at birth to $\sim 36 \mathrm{~mm}$ in adults, and reached $90 \%$ of adult value by $\mathrm{P} 42$. The pinnae dimensions, quantified by the effective diameter, were $\sim 58 \%$ smaller than adult at birth and more than doubled in size during development. The pinnae reached maturity by $\sim \mathrm{P} 45$. Based on the growth functions and the distribution of adult values (shaded areas), maturation of the head and pinnae are complete by $\sim \mathrm{P} 42-45$ or $6-7$ weeks after birth.

\section{Development of monaural acoustical transformations}

Spatial distribution of DTF amplitude gain. The DTF gain at a given frequency varied with source direction and with the age of the animal. Figure 2A shows the distribution of DTF gains for 2, 4, 8, 10, 12, 16, and $20 \mathrm{kHz}$ (upper right, right column) for sources in the frontal hemispheres for the right ear. Each of the gain plots was normalized by the maximum DTF gain (upper left side of each panel) observed at that frequency and contours encompassing multiples of $-3 \mathrm{~dB}$ of the maximum gain were plotted. For a given frequency, the maximum gain tended to increase and the spatial area of increased gain bounded by the $-3-\mathrm{dB}$ contour tended to decrease with the age of the animal. For reference, Figure 2B illustrates a schematic representation of the speaker positions for the gain plots (Fig. 2A) in reference to the recordings taken from the right ear, i.e. the maximum gain for the right ear would be generated for a speaker position on the right.

To further illustrate the findings in Figure 2A, Figure 2C plots the maximum gain in the DTFs (which does not include the ear canal and concha resonances) as a function of frequency for animals of three different ages (the $\mathrm{P} 0-1$ and Adult data are from Fig. 2A). In all animals, the maximum gain tended to increase with the frequency, at least up to $\sim 10-20 \mathrm{kHz}$. However, for frequencies $<10 \mathrm{kHz}$ there was a systematic increase of maximum gain with age. The gain curves appear to be simply shifted toward lower frequencies with age, consistent with the increase in the linear dimensions of the head and pinnae (Fig. 1). Regardless of age, a gain of $\sim 3-$ $5 \mathrm{~dB}$ was observed at low frequencies $<\sim 3 \mathrm{kHz}$ and far lateral angles (e.g., Fig. 2A) and was likely due to the socalled obstacle effect of the head (Kinsler et al. 1982). 
A

P0-1

$3 \mathrm{~dB}$

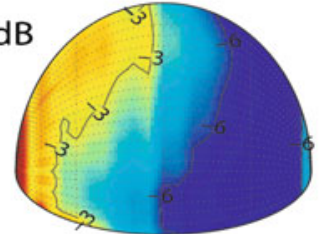

$4 \mathrm{~dB}$

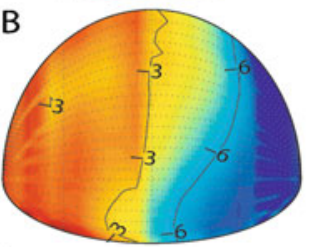

$10 \mathrm{~dB}$

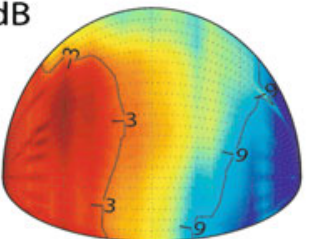

$8 \mathrm{~dB}$

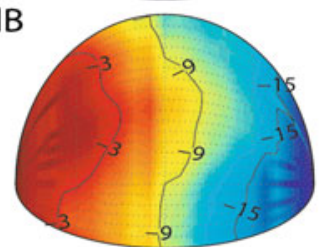

$5 \mathrm{~dB}$

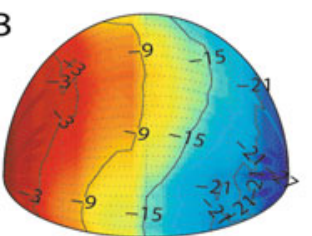

$15 \mathrm{~dB}$

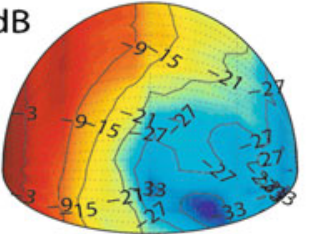

$15 \mathrm{~dB}$

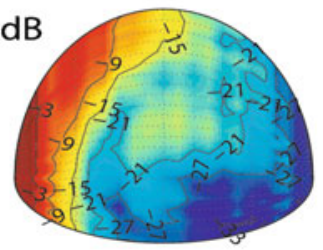

$10 \mathrm{~dB}$

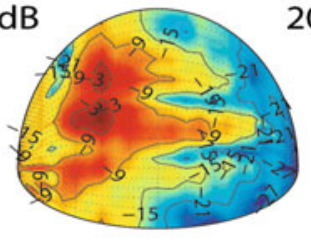

$10 \mathrm{~dB}$

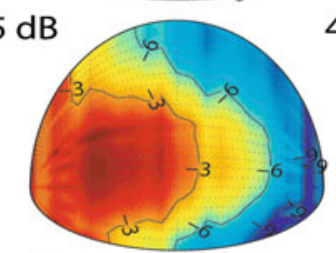

$10 \mathrm{~dB}$

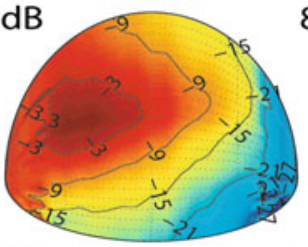

$10 \mathrm{~dB}$
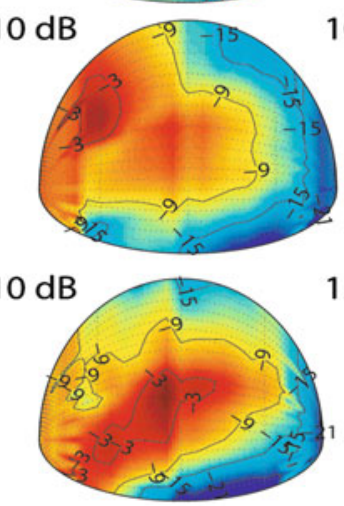

$15 \mathrm{~dB}$

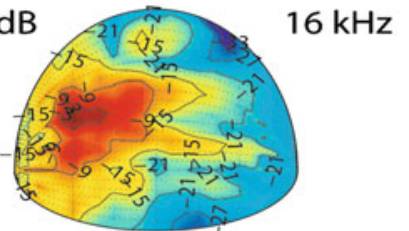

$20 \mathrm{kHz}$

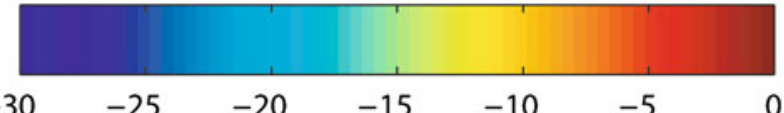

B

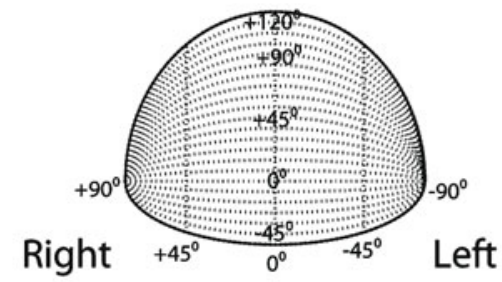

C

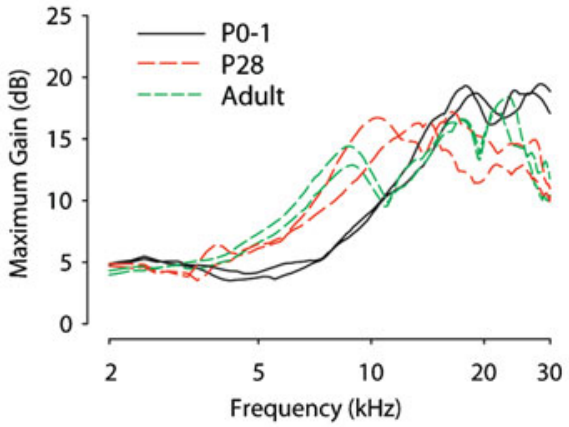

D

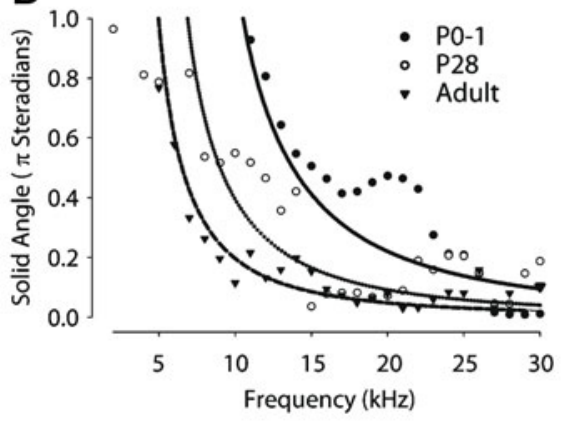

E

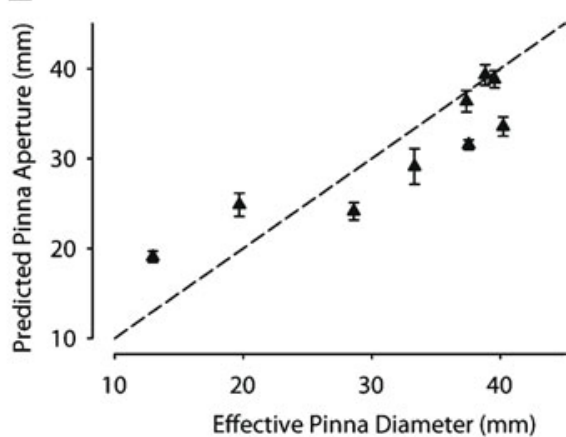

FIG. 2. Spatial distribution of DTF gains for seven frequencies for the right ears (e.g., $+90^{\circ}$ is ipsilateral) of two animals, aged P0-1 and adult. A DTF gains for each animal have been normalized by the maximum gain (indicated at the upper left of each panel) at the indicated frequency (upper right of panels in last column). The contours are plotted at $-3-\mathrm{dB}$ intervals from the maximum gain. B Illustration of the loudspeaker positions for the gain plots (A) in reference to the recordings taken from the right ear, i.e. the maximum gain for the right ear would be generated for a speaker position on the right, shown in red. C Maximum acoustical gain of the head and pinna as a function of frequency for animals aged P0-1, P28, and adult. D Solid area (in units of $\pi \mathrm{sr}$ ) enclosed by the $-3-\mathrm{dB}$ contour from the DTF gain plots (A) as a function of frequency. Solid lines indicate the best-fitting circular aperture model (see text) to the data corresponding to each animal. E Scatter plot of the predicted aperture diameter from the circular aperture model fitted to data as a function of the empirically measured pinnae dimension in nine animals. Error bars show $95 \%$ confidence intervals. Dashed line indicates line of equality. 
The spatial distributions of DTF gain (Fig. 2A) were quantified by the directionality of the pinnae and head obtained by computing the solid angle contained by the $-3-\mathrm{dB}$ contour at frequencies from 2 to $30 \mathrm{kHz}$ in $1 \mathrm{kHz}$ frequency steps. Figure 2D shows the $-3-\mathrm{dB}$ contour area (in $\pi$ steradians (sr); here $2 \pi$ sr equals $1 / 2$ hemisphere) as a function of frequency for right ears for the three animals of different ages. For frequencies $\leq 4-5 \mathrm{kHz}$, the area covered more than one hemisphere ( $2 \pi \mathrm{sr}$, not shown in figures). As shown graphically in Figure 2A, at higher frequencies, the $-3-\mathrm{dB}$ contour area systematically decreased with frequency for all animals. We hypothesized that for a given frequency, the solid area enclosed by the $-3-\mathrm{dB}$ contour should decrease with the age of the animal because the linear dimensions of the external ear increase during development. To test this hypothesis, the equation describing the frequency dependence of the solid angle for a $-3-\mathrm{dB}$ contour for acoustic diffraction through a circular aperture for a given aperture diameter (equation derived in Calford and Pettigrew (1984), and Coles and Guppy (1986); see also Tollin and Koka (2009b)) was fitted to the acoustical data for nine animals (only those animals for which we measured DTFs at the full 525 locations were used here) at different ages ranging from $\mathrm{P} 0-1$ to adult. In fitting the aperture, diameter was the only free parameter (MATLAB $^{\circledR}$ Version 7.1 robust nonlinear least squares trust-region method). For some animals, we noticed that the empirical data at lower frequencies were poorly fitted by the model, so only data for solid angles $\leq 0.5$ were used for the fitting resulting in frequencies $\geq 5-15 \mathrm{kHz}$ depending on the age of the animal, although all empirical data are shown in Figure 2D. The best-fitting functions describing the solid angle are shown for three animals in Figure 2D; the predicted diameters of the circular aperture for these animals were 19.0, 24.1, and $39.3 \mathrm{~mm}$, respectively $\left(R^{2}\right.$ of the fits were $0.94,0.76$, and 0.96, respectively). Coefficients of determination $\left(R^{2}\right)$ for the population averaged $0.84 \pm 0.09$ with a median of 0.8 and a range of $0.7-0.96$.

To test the hypothesis that the increasing linear dimensions of the pinnae during development determine the directionality as computed by the $-3-\mathrm{dB}$ area as a function of frequency (e.g., Fig. 2D), the predicted pinnae aperture from the fitted function was plotted in Figure 2E as a function of the empirical effective diameter (as described in Fig. 1B) of the pinnae. The linear regression (not shown; dashed line indicates line of equality) of predicted pinnae aperture diameter on the effective diameter was significant $(R=0.91 ; P=0.01 ; n=9)$ and resulted in an equation of the form $y=10.5+0.6 x$. These data support the hypothesis that the linear dimensions of the pinnae, in particular, the effective diameter, determine the devel- opment of the frequency dependence of the $-3-\mathrm{dB}$ area of the acoustical directivity.

Acoustical axis. For a given frequency, the direction of the maximum gain occurs at the acoustic axis (Middlebrooks and Pettigrew 1981; Phillips et al. 1982) and the spatial location of this acoustic axis varied as a function of frequency. Figure 3 shows the spatial location of the acoustic axis for elevation (top) and azimuth (bottom) as a function of frequency for one ear in animals of three different ages. For source elevation, at all ages the acoustic axis tended to increase in elevation with increases in frequency that were then interrupted by transitions to lower elevations followed by increases in elevation again for further increases in sound frequency (for example, see green squares, Fig. 3). The first of these acoustic axis transitions in source elevation for each age is marked by a vertical line of the same color as the age group indicated above the plots. The data show that there was a systematic decrease in the frequency range at which the first transition occurs. The shifts in the transitions of the acoustic axes as a function of development coincides with the shift in the first notch frequency ranges discussed below. The location of the acoustic axis in azimuth had a
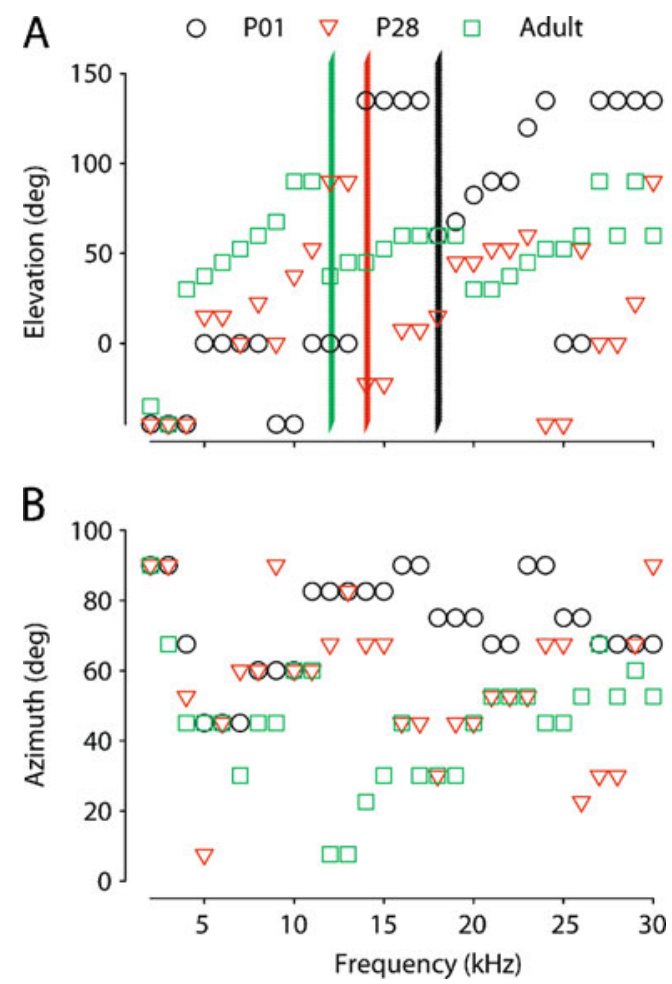

FIG. 3. The elevation (A) and azimuth (B) corresponding to the acoustic axis as a function of frequency for animals aged P0-1, P28, and adult. Vertical lines in $(\mathbf{A})$ indicate frequencies where discrete transitions appear to occur in the acoustic axes in sound source elevation. 
more complicated pattern and was therefore more difficult to interpret.

Frequency range and spatial-location dependence of broadband spectral notches. Systematic changes were observed in the frequency of the first (i.e., lowest frequency) broadband spectral notch (i.e., first notch frequency (FNF), (Rice et al. 1992)) with changes in source location in the frontal hemisphere in animals of all ages. The FNF tended to increase in animals of all ages as the sound source position moved up in elevation and toward the ipsilateral ear. The depths of the spectral notches were $\sim 10-15 \mathrm{~dB}$ in the youngest animals and were $\sim 15-20 \mathrm{~dB}$ in adults. The FNF also decreased from higher frequencies $(\sim 17 \mathrm{kHz})$ in animals aged P0-1 to lower frequencies $(\sim 6.5 \mathrm{kHz})$ as seen in adult animals. Figure $4 \mathrm{~A}-\mathrm{C}$ shows the DTFs for the right ears of three animals of different ages (P0-1, P28, and adult) for elevations ranging from $-30^{\circ}$ to $45^{\circ}$ in $15^{\circ}$ steps at $0^{\circ}$ azimuth. For a given source location, the FNF also tended to decrease with age (Fig. 4A-C). For example, for a sound source directly in front of the animal $\left(0^{\circ}\right.$, $\left.0^{\circ}\right), \mathrm{FNFs}$ were $17,9.5$, and $6.5 \mathrm{kHz}$ for animals aged P0-1, P14, and adult, respectively. These developmental aspects of the FNF are illustrated in Figure 4D, where FNFs at $0^{\circ}$ azimuth and changing elevation for animals at six ages are shown. At all ages, FNFs increases with elevation; however, the absolute ranges of FNFs shifts to progressively lower frequencies during development.
To test the hypothesis that the developmental change in $\mathrm{FNF}$ range is determined by the development of pinnae size, Figure $4 \mathrm{E}$ shows the FNF for a single source location $\left(0^{\circ}, 0^{\circ}\right)$ as a function of the pinnae effective diameter in 19 animals ( $n=38$; 2 pinnae per animal); the error bars show the overall range of detectable FNFs observed in the frontal hemisphere for the 11 younger animals used in this studies. The remaining data points for adults had FNFs encompassing a range from $\sim 5.5$ to $19 \mathrm{kHz}$ (shaded area, Fig. $4 \mathrm{E}$ ). The data show that FNFs did not consistently encompass or fully fall within the adult range in all animals until animals reached $\sim 6$ weeks of age (Fig. 4D and E). Recall in Figure $1 \mathrm{~B}$ that the effective diameter of the pinnae dimension reached $90 \%$ of adult size, or $\sim 34 \mathrm{~mm}$, by P42. Figure $4 \mathrm{E}$ shows that the ranges of FNFs observed in animals with pinnae dimensions $>34 \mathrm{~mm}$ were all adult like while those $<34 \mathrm{~mm}$ generally were not. A threeparameter exponential decay function (not shown) accurately describes the development of the FNF at $0^{\circ}$ as a function of the effective diameter of the pinnae $\left(R^{2}=0.76 ; P<0.0001\right.$; and $\left.y=8.76+54.48 e^{-0.125 x}\right)$ supporting the hypothesis that the linear dimensions of the pinnae determine the FNFs. In adult animals, pinnae removal eliminated the spectral notches in both ears (not shown; the contribution of the pinnae to the cues to location in chinchilla will be described in detail in a separate paper). The spectral notch cues achieved
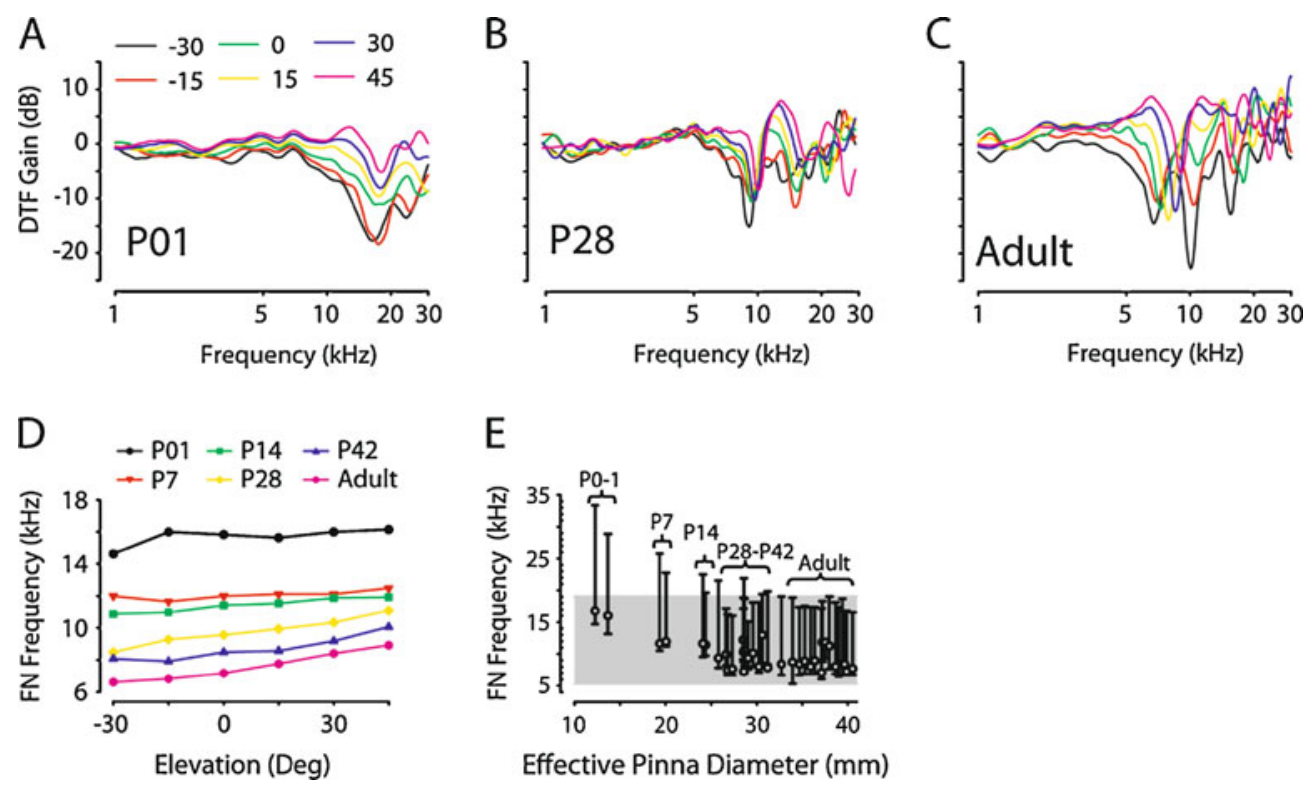

FIG. 4. Development of the monaural broadband spectral notch cues. A-C DTF gain for seven different elevations from $-30^{\circ}$ to $+45^{\circ}$ at $0^{\circ}$ azimuth for chinchillas of different ages (lower left of panel). D FNFs plotted as a function of elevation at $0^{\circ}$ azimuth for animals of six different ages. E Development of the first notch frequency range as a function of the development of effective pinna diameter $(n=38$ pinnae). Symbols indicate $\mathrm{FNF}$ at $\left(0^{\circ}, 0^{\circ}\right)$ while the error bars indicate the range of detectable FNFs observed in the frontal hemisphere. Gray-shaded area indicates the range of FNFs observed in the frontal hemisphere in adult animals. 
adult-like status by at least 6 weeks (P42), consistent with the development of the dimensions of the pinnae themselves as illustrated in Figure 1B.

\section{Development of the binaural cues to sound location}

\section{Interaural level differences}

Spatial distribution of ILDs. The ILD spectra (i.e., ILD as a function of frequency for a given source location) results from the difference between left and right ear DTF gains. The magnitudes of the ILD cues varied with frequency, source location and with the age of the animals. Positive and negative ILDs indicate higher DTF gain for right and left ears, respectively. The spatial distributions of ILD cues computed from 2 to $20 \mathrm{kHz}$ are shown (Fig. 5, upper right of right-hand column) for a P0-1 and an adult animal. Frequencies higher than $20 \mathrm{kHz}$ produced complicated plots of ILD. All plots were normalized appropriately and share the common scale bar to indicate the value of ILD in decibels (Fig. 5, bottom color bar). The maximum ILD for each animal and frequency is indicated at the upper left-hand side of each plot. The maximum ILDs tended to increase with increasing frequency across all ages. A major developmental trend was observed regarding ILDs for any given source location, that maximum ILDs increased with animal age for frequencies $<12 \mathrm{kHz}$. This developmental trend is explored next.

Spatial and frequency distributions of ILDs along horizontal plane. Interpreting ILD spatial plots (Fig. 5) over fine spatial position and frequency increments can be difficult. Here, since the ILD is likely to be most useful for sound localization of sources along the azimuth plane, Figure 6 plots the ILD as a joint function of frequency and source azimuth for sources along the horizontal plane (i.e., $0^{\circ}$ elevation). These functions are shown for three animals of different ages: P0-1 (A), P14 (B), and adult (C). Across all ages, ILDs are complex functions of azimuth and frequency. ILDs vary little along the azimuth for lower frequencies; however, at higher frequencies, ILDs can vary as much as $\pm 35-40 \mathrm{~dB}$ in adults. General trends were observed concerning the development of ILD cues. For example, as the animals aged, for a given frequency, the ILDs increased in magnitude and the overall ILD versus azimuth functions shifted toward lower frequencies.

Figure 6D illustrates the systematic shift of the ILD spectra at $75^{\circ}$ azimuth and $0^{\circ}$ elevation for all age groups. Over the range of frequencies plotted, the maximum ILD at $\mathrm{P} 0-1$ for this source location was
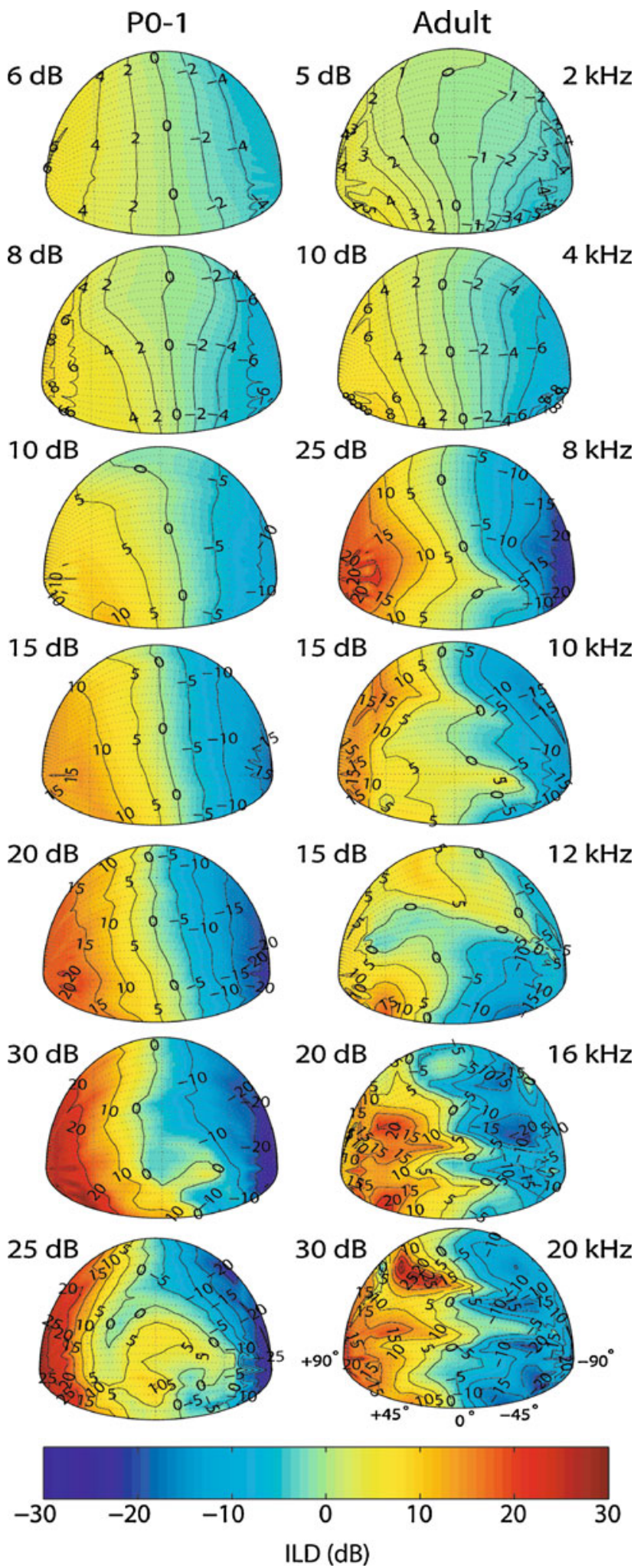

FIG. 5. Spatial distributions of ILD for seven frequencies (upper right, right column) in two animals aged P0 and adult. Maximum ILD for each animals and each frequency is indicated at the upper left-hand side of each plot. Color bar indicates the ILD in decibels. 


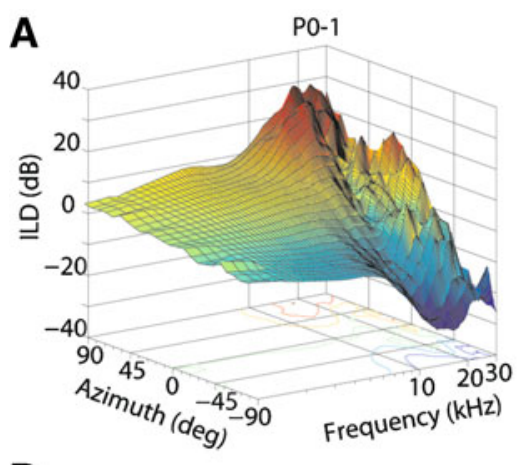

D

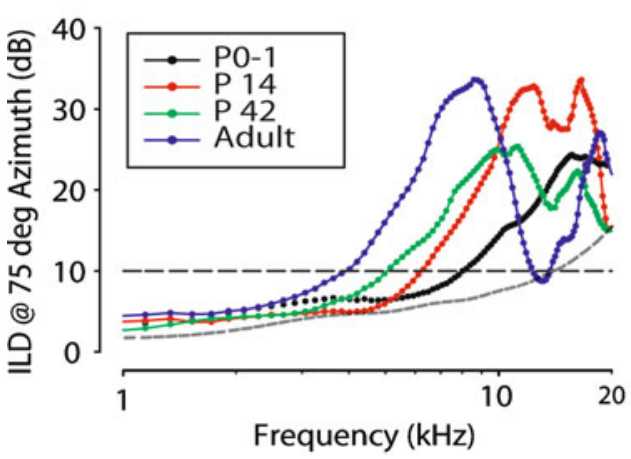

FIG. 6. Spatial and frequency distributions of ILDs along the horizontal plane $\left(0^{\circ}\right.$ elevation) for animals aged P0-1 (A), P14 (B), and adult (C). D ILD spectrum at $75^{\circ}$ azimuth for four animals aged P0 through adulthood. The curved dashed line shows the ILD spectrum at $75^{\circ}$ azimuth predicted by the spherical head model of Duda and Martens (1998) using an adult chinchilla head diameter. E
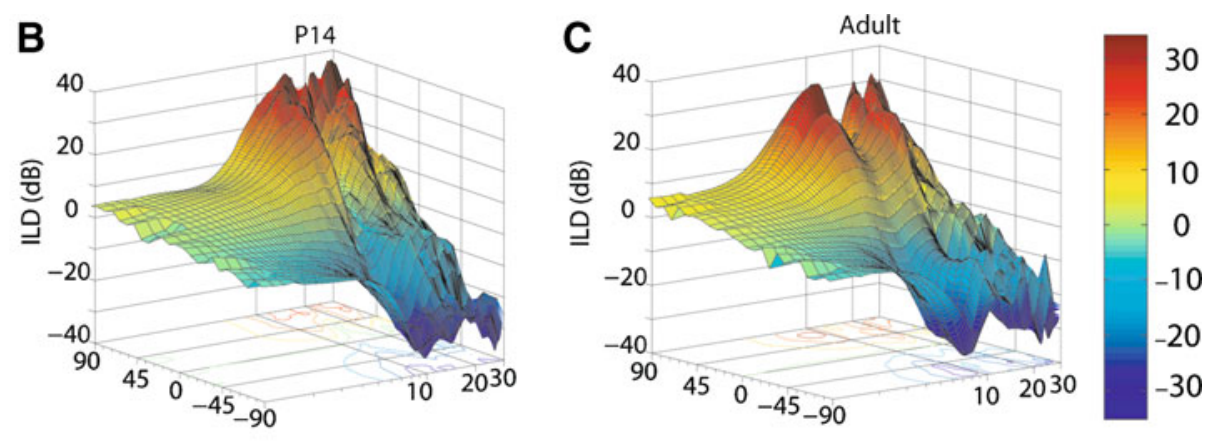
10 0 $-30$
E

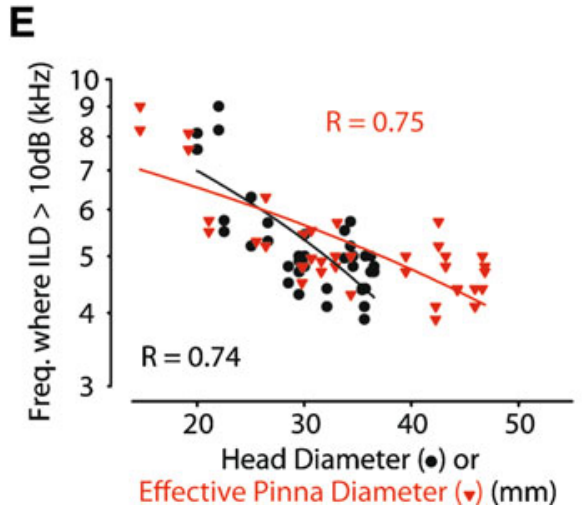

Frequency where the ILD cue first reached $10 \mathrm{~dB}$ for a source at $75^{\circ}$ azimuth (as in (D)) as a function of both head radius (circles) and pinna height (triangles). Pinnae size data for each animal are plotted for both the left and right ears. Solid lines indicate the linear regression through the respective data points. The correlation coefficients are also indicated for the regression. $\sim 25 \mathrm{~dB}$, occurring at nearly $20 \mathrm{kHz}$. In contrast, in the older animals, maximum ILDs at this location reached $\sim 35 \mathrm{~dB}$ and occurred at much lower frequencies. Note that in each plot of the ILD spectrum for each animal for this location, there is an inflection point where the magnitude of the ILD begins to increase rapidly with changes in frequency. The frequency of this inflection point systematically decreased with increasing developmental age. To capture this finding, in each plot of the ILD spectrum, a particular value of ILD $(10 \mathrm{~dB})$ is indicated by a horizontal line. The frequency at which this $10 \mathrm{~dB}$ ILD metric occurred was measured in each animal for a source located at $\left(75^{\circ}, 0^{\circ}\right.$; note that similar types of changes were observed for other azimuthal locations). Tollin and Koka (2009a) showed in the cat that a systematic shift in the frequency range of this $10 \mathrm{~dB}$ ILD metric (i.e., the frequency at which the ILD first reaches $10 \mathrm{~dB}$ ) occurs for all locations between midline and $90^{\circ}$ with the largest shifts occurring at $90^{\circ}$.

Growth of head and pinnae contributes to the increase in magnitude and frequency range of ILDs. It has been shown in the cat that the measured ILDs are considerably different from the predictions computed from a spherical head model across various ages of development
(Tollin and Koka 2009a). Here we also show that the ILD prediction from the spherical head model of Duda and Martens (1998) with an adult head diameter of $35.5 \mathrm{~mm}$ (dashed line, Fig. 6D) vastly underestimates the actual ILD magnitudes experienced by the chinchilla particularly for mid- to high-frequencies. Excluding head diameter, the pinnae are the only other structures on the head that could account for these differences. In order to test this hypothesis that ILDs are generated by both the head and pinna, Figure $6 \mathrm{E}$ plots the frequency at which the ILD first exceed $10 \mathrm{~dB}$ at a location of $+75^{\circ}$ azimuth and $0^{\circ}$ elevation (as plotted in Fig. 6D) as a function of both head diameter and effective pinnae diameter (i.e., Fig. 1B). This ILD factor was significantly correlated with both head ( $r=-0.74 ; p<$ 0.0001 ; and $n=19)$ and pinnae $(r=-0.74 ; p<0.0001$; and $n=38$ pinna) dimensions. For both dimensions, the frequency at which the ILD reached $10 \mathrm{~dB}$ decreased systematically as the head and pinnae increased. However, the fact that the empirical ILDs vastly exceed those produced by a spherical head model with adult dimensions suggests that the pinnae themselves were the predominant determinants of the developmental changes in ILD, at least over the range of frequencies examined here. 


\section{Interaural time differences}

Spatial distribution of ITDs. The spatial distributions of low-frequency $(<3.5 \mathrm{kHz})$ ongoing ITDs are shown in Figure 7A for animals aged P0-1, P14, and adult. Across all ages, the ITDs increased with changes in source azimuth away from the midline, but for a given source azimuth the ITDs were relatively constant with changes in elevation (i.e., the contour lines are vertically oriented, parallel with iso-sourceazimuth contours). The magnitudes of the ITDs also increased with age for a given source azimuth. This trend is illustrated more plainly in Figure 7B which shows ITD magnitude as a joint function of azimuth along the horizontal plane $\left(0^{\circ}\right.$ elevation $)$ for animals at various ages. Symbols represent across-animal mean ITD magnitude with the standard deviation as error bars. The data in Figure $7 \mathrm{~B}$ demonstrates that the magnitudes of the ITDs for any source azimuth systematically increase with animal age.

Increasing head diameter contributes to the developmental increase in magnitude ITDs. The systematic increase in ITD magnitude with increasing animal age is hypothesized to be a result of the increase in the head diameter during development. To test this, Figure $7 \mathrm{C}$ plots the maximum ITD as a function of head diameter. Maximum ITDs increased systematically on average from $\sim 160$ to $280 \mu \mathrm{s}$, a factor of 1.75 as the head increased on average from 19.8 to $35.5 \mathrm{~mm}$, a factor of 1.80. The linear regression of maximum ITD on head diameter was significant $(R=0.872 ; p<0.0001$; $y=8.98+7.59 x, n=19)$. As expected, head diameter directly correlated with the magnitude of the ITD cue to source location. However, it has been suggested by Koka et al. (2008) and Tollin and Koka (2009a) that the pinnae can also contribute to ITDs. One explanation for the role of the pinnae in ITD production, as suggested by Koka et al. (2008), is that a considerable portion of the pinnae and the distal parts of the auditory meatus in mammals such as the cat and rat that have ear canals formed from cartilage remains in the path of the sound. Thus, the pinnae may function to make the acoustically effective diameter of the head larger, thus increasing the magnitude of the ITDs. Consistent with this hypothesis, the predicted maximum ITD based on the empirically measured head radii and the spherical head model of Kuhn (1987) for low-frequency ongoing ITDs (Fig. 7C, gray line) was $\sim 25 \%$ less than the empirically measured ITDs. This discrepancy may result from the increased ITD due to the pinnae.
A

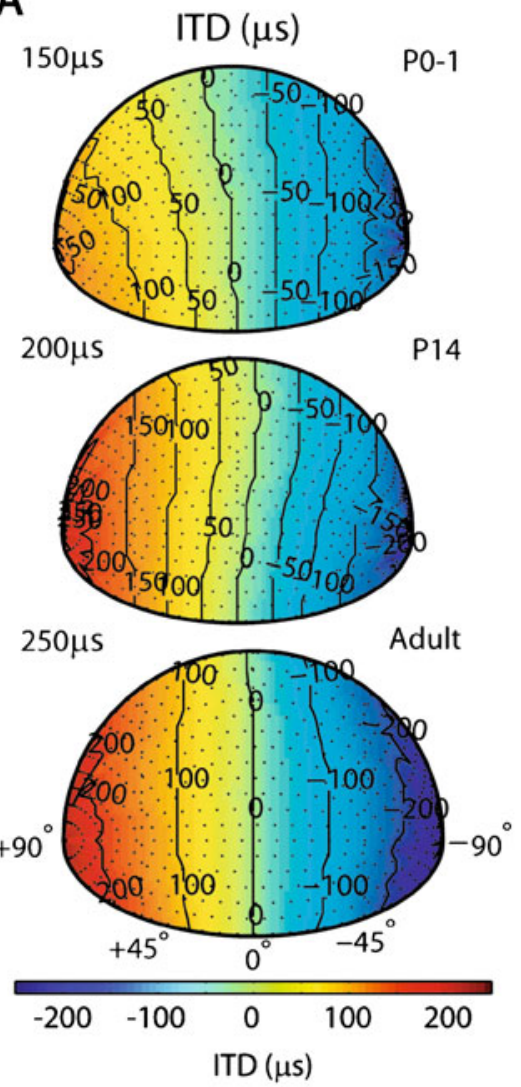

B
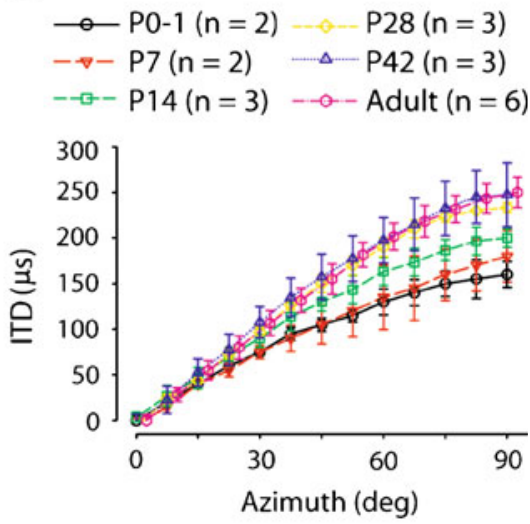

C

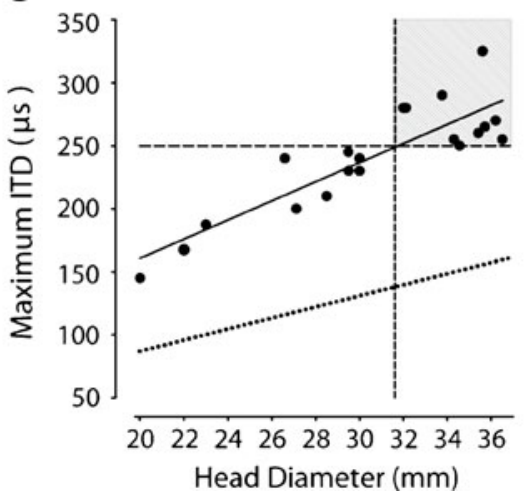

FIG. 7. A Spatial distributions of the ITD for animals aged P0-1, P14, and adult. B ITD as a function of source azimuth along the horizontal plane $\left(0^{\circ}\right.$ elevation $)$ for animals aged P0 through adulthood (age given by the parameter; adult data points have been shifted by $2.5^{\circ}$ to differentiate them visually from the P42 data). C Maximum ITD as a function of head diameter ( $n=19$ animals). Shaded region indicates ranges of maximum ITDs and head radii in adults. Solid line indicates the linear regression of maximum ITD on head radius. The gray line indicates the ITD prediction from the spherical head model of low-frequency ITDs proposed by Kuhn (1987) for the given head diameter. 


\section{DISCUSSION}

Many attributes make the chinchilla an attractive model for anatomical, physiological and behavioral studies of the binaural auditory system. Among the mammals behaviorally tested for sound localization acuity, the chinchilla ranks as one of the best rodents (Heffner et al. 1994). In addition to sound localization, chinchillas can perform complex auditory psychophysical tasks (Shofner et al. 2007) similar to humans. Chinchillas are also widely used for experimentally induced otitis media research (Margolis et al. 1995; Giebink 1999). Aside from studies in the cat (Moore and Irvine 1979; Tollin and Koka 2009a, b), ferret (Schnupp et al. 2003), and barn owl (Knudsen et al. 1984a; Knudsen et al. 1984b), there have been few studies on the development of the physical dimensions of the head and pinna and the changes in the resulting localization cues.

Development of the head and pinnae determine the development of the cues to sound location

\section{Monaural cues}

The maximum monaural acoustical gain produced by the head and pinna increased with frequency and age. Across all ages, a gain of $\sim 3-5 \mathrm{~dB}$ was observed at low frequencies $<\sim 3 \mathrm{kHz}$. At higher frequencies, the maximum gains increased, reaching values of $10-$ $15 \mathrm{~dB}$ for both younger and adult animals (Fig. 2A). The adult data agree with previous reports for chinchilla (von Bismark 1967; Murphy and Davis 1998). Although maximum gains were similar between all ages, there were differences in the DTF gain directionality, its frequency dependence, and its development. We tested the hypothesis that the increases in acoustic directionality during development were determined by increasing pinnae dimensions by modeling the pinnae as a circular aperture (Calford and Pettigrew 1984; Coles and Guppy 1986) and then comparing the outputs of this model to the empirical acoustical measurements. Our results fit well with previous studies showing that the frequency dependence of diffraction of sound into a circular aperture accounts qualitatively for the area of acoustic directionality (Calford and Pettigrew 1984; Coles and Guppy 1986; Carlile and Pettigrew 1987; Musicant et al. 1990; Obrist and Wenstrup 1998; Tollin and Koka 2009b). Our data demonstrate that the increasing dimensions of the pinnae during development were accompanied by increased acoustical directionality.

The acoustic axis for sources in azimuth was complicated and difficult to interpret. However, in animals of all ages, the acoustic axis for source elevation migrates from low to high elevations with increasing frequency, then at a certain frequency jumps back to lower elevations and again increases in elevation (e.g., Fig. 3). The frequencies at which these transitions occurred shifted from higher frequencies $(\sim 18 \mathrm{kHz})$ for $\mathrm{P} 0-1$ animals to lower frequencies $(\sim 12 \mathrm{kHz})$ in mature adults. These general trends in the movement in the acoustic axis in adult animals are similar to those reported in other species [cat (Musicant et al. 1990), wallaby (Coles and Guppy 1986), ferret (Carlile 1990), rats (Koka et al. 2008), and bats (Firzlaff and Schuller 2003)].

Across all ages, the FNF (Rice et al. 1992) systematically increased from lower to higher as sound source elevation increased and moved toward the ipsilateral ear. However, the frequency ranges of FNFs were dependent on age, with FNFs ranging from 14.7-33.4 kHz at P0-1 to $5.3-19.1 \mathrm{kHz}$ in adults. This means that the FNF associated with a particular source location systematically decreased with age, across all source locations. The data demonstrate a developmental decrease of the FNFs and their ranges that is likely due to the increase in the size of the pinna (Fig. 4E). This trend is consistent with the hypothesis that an increased-size pinna will interact with sounds of progressively lower frequencies (see Tollin and Koka 2009b). FNFs fall fully into the adult range by $\sim \mathrm{P} 45$, corresponding to when the pinna dimensions become mature. Effective pinna diameters begin to fall into the adult range at $\sim \mathrm{P} 45$ with a value of $34 \mathrm{~mm}$ (Fig. 1). The range of FNFs fall into the adult range for pinna sizes $>34 \mathrm{~mm}$ (Fig. 4B) but fall outside the range for smaller pinnae sizes. This fact and the shift in frequency ranges of the FNFs observed as the pinnae grow demonstrates that the pinna dimensions directly determines the functional range of FNFs during development and their final range as adults.

\section{Binaural cues}

ILDs were dependent on both frequency and spatial location. For frequencies $<5 \mathrm{kHz}$, ILDs were small but rapidly increased for higher frequencies and systematically varied with azimuth. In order to test the hypothesis that the development of the pinnae dimensions contribute to the development of ILD cues, the $+10-\mathrm{dB}$ ILD metric was plotted as a function of both head diameter and pinna effective diameter (Fig. 6E). There was a correlation of both head diameter and pinna height to the decrease in the frequency at which the first ILD of $10 \mathrm{~dB}$ occurs. This result, along with the empirical observations that the ILD metrics reach adult-like status by P42, fits well with the empirical head and pinna growth measurements showing adult-like size by $\sim 6$ weeks postnatal. 
The ITD cue depends mostly on sound source azimuth. The maximum ITDs increased by a factor of 1.75 as the head diameter increased by a factor of 1.80. We also observed low-frequency ongoing ITDs to be $\sim 1.25$ times larger than the ITDs predicted by a theoretical spherical head model (Kuhn 1987) of lowfrequency ITDs (low frequency defined here as $<3.5 \mathrm{kHz}$ ). Differences between predicted ongoing ITDs from spherical head models and empirical ITDs have been previously reported (cat (Roth et al. 1980) (Tollin and Koka 2009a); monkey (Spezio et al. 2000; Slee and Young 2010); gerbil (Maki and Furukawa 2005); guinea pig (Sterbing et al. 2003); rat (Koka et al. 2008); rabbit (Kim et al. 2010)). In the rat, Koka et al. (2008) demonstrated that the pinnae contribute to the largerthan-modeled ITDs. We have shown this in the chinchilla and the data are in a concurrent publication. The data indicates that the chinchilla begins to experience adultlike ITD magnitudes by $\sim$ P42. Grouping the maximum ITDs and associated head diameters (shaded area, Fig. 7C) indicates that a head diameter of $\sim 32 \mathrm{~mm}$ is required to reach adult-like ITDs of $250 \mu$ s or greater.

\section{Comparison to human development}

The goal of animal research is to explore how systems in other species work in hope that the knowledge gained might be applied to solving problems observed in the human condition. In order to establish a more accurate comparison to human development, with regards to head diameter (and the associated acoustic cues to location), Table 1 was generated from averaged data from three studies reported for human interaural distance (Davenport 1940) coinciding nicely with another report of the same nature (Tilley 1993). The percent of full head growth and the age correlating to the percentage for both human and chinchilla are reported here. For example, a human head is $75 \%$ of the final mature head size by

\begin{tabular}{lcccc}
\hline \multicolumn{5}{c}{ TABLE 1 } \\
\hline \multicolumn{5}{c}{ Comparison of chinchilla and human head growth } \\
\hline $\begin{array}{l}\% \text { of Adult } \\
\text { head size }\end{array}$ & $\begin{array}{l}\text { Wuman head } \\
\text { width (mm) }\end{array}$ & $\begin{array}{l}\text { Human age } \\
\text { (months) }\end{array}$ & $\begin{array}{l}\text { Chinchilla head } \\
\text { width }(\mathrm{mm})\end{array}$ & $\begin{array}{l}\text { Chinchilla } \\
\text { age (days) }\end{array}$ \\
\hline $70 \%$ & $98.5 \pm 1.96$ & $\sim 1$ & $24.41 \pm 1.90$ & $\sim 7$ \\
$75 \%$ & $107.8 \pm 2.29$ & $\sim 3$ & $26.15 \pm 2.18$ & $\sim 14$ \\
$80 \%$ & $114.4 \pm 3.9$ & $\sim 4$ & $27.9 \pm 1.89$ & $\sim 24$ \\
$85 \%$ & $121.13 \pm 2.87$ & $\sim 7$ & $29.64 \pm 2.18$ & $\sim 28$ \\
$90 \%$ & $128.25 \pm 3.04$ & $\sim 12$ & $31.38 \pm 2.12$ & $\sim 35$ \\
$95 \%$ & $135.36 \pm 4.17$ & $\sim 30$ & $33.13 \pm 3.46$ & $\sim 42$ \\
\hline
\end{tabular}

The percentage of adult head size and the approximate time this occurs for both human and chinchilla is shown. The acoustical cues to sound location have been shown here, and in previous work, to be correlated with developmental increases in head diameter. Human data was generated from Davenport (1940). $\sim 3$ months and for the chinchilla it is $\sim \mathrm{P} 14$ so as far as the development of the interaural distance is concerned, a P14 chinchilla coincides with a 3 month old human. The purpose of Table 1 is to establish a comparable developmental timeline between human and chinchilla that can be used for research on the effects of experimentally induced conductive hearing loss (i.e., simulated otitis media) on the anatomical, neurophysiological, and behavioral mechanisms of sound localization.

\section{Implications for physiological and behavioral development}

The chinchilla is a popular model for anatomical, physiological, behavioral studies of audition. The data presented here are relevant to these studies on the basis that the general development of the auditory system is influenced to a large degree by the development of the acoustical properties of the head and pinna. The cues to location have been shown to be established by the transformations to the sound waves propagating from a source by the head and pinna (Kuhn 1987). Understanding how the cues themselves change as a result of the developmental increase in the physical dimensions of these structures, should further our knowledge of how the neural encoding of these cues would develop and ultimately how the animal uses both for the perception of sound location throughout development.

\section{ACKNOWLEDGMENTS}

This work was supported in part by the National Institutes of Deafness and Other Communicative Disorders Grant DC006865 to DJT and by the Basic Neuroscience Anatomy Training (BNAT) Grant T32NS007083 to HGJ. We appreciate the assistance of Dr. Michael Hall for constructing custom experimental equipment (with support by NIH grant P30 NS041854-05).

\section{REFERENCES}

Alkhatib A, Biebel UW, Smolders JW (2006) Inhibitory and excitatory response areas of neurons in the central nucleus of the inferior colliculus in unanesthetized chinchillas. Exp Brain Res 174:124-143

Bugayevskiy LM, Snyder JP (1995) Map projections: a reference manual. Taylor \& Francis, London

Calford MB, Pettigrew JD (1984) Frequency dependence of directional amplification at the cat's pinna. Hear Res 14:13-19

Carlile S (1990) The auditory periphery of the ferret. II: The spectral transformations of the external ear and their implications for sound localization. J Acoust Soc Am 88:2196-2204

Carlile S, Pettigrew AG (1987) Directional properties of the auditory periphery in the guinea pig. Hear Res 31:111-122 
Chen GD, Sinex DG (1999) Effects of interaural time differences on the responses of chinchilla inferior colliculus neurons to consonant-vowel syllables. Hear Res 138:29-44

Coles RB, Guppy A (1986) Biophysical aspects of directional hearing in the tammar wallaby, Macropus Eugenii. J Exp Biol 121:371-394

Davenport CB (1940) Post-natal development of the head. Proc Am Philos Soc 83:1-215

Duda RO, Martens WL (1998) Range dependence of the response of a spherical head model. J Acoust Soc Am 104:3048-3058

FirzlafF U, Schuller G (2003) Spectral directionality of the external ear of the lesser spear-nosed bat, Phyllostomus discolor. Hear Res 181:27-39

Giebink GS (1999) Otitis media: the chinchilla model. Microb Drug Resist 5:57-72

GiLBERT SG (1981) Pictorial anatomy of the cat. University of Washington Press, Seattle

Harrison RV, Kakigi A, Hirakawa H, Harel N, Mount RJ (1996) Tonotopic mapping in auditory cortex of the chinchilla. Hear Res 100:157-163

Heffner RS, Heffner HE (1991) Behavioral hearing range of the chinchilla. Hear Res 52:13-16

Heffner RS, Heffner He, Kearns D, Vogel J, Koay G (1994) Sound localization in chinchillas. I: left/right discriminations. Hear Res $80: 247-257$

Heffner RS, Heffner HE, KoAy G (1995) Sound localization in chinchillas. II. Front/back and vertical localization. Hear Res 88:190-198

Heffner RS, Koay G, Heffner HE (1996) Sound localization in chinchillas, III: effect of pinna removal. Hear Res 99:13-21

Kim DO, Bishop B, Kuwada S (2010) Acoustic cues for sound source distance and azimuth in rabbits, a racquetball and a rigid spherical model. J Assoc Res Otolaryngol. doi:10.1007/510162010-0221-8

Kinsler LE, Frey AR, Coppens AB, Sanders JV (1982) Fundamentals of acoustics. Wiley, New York

Knudsen EI, Esterly SD, Knudsen PF (1984a) Monaural occlusion alters sound localization during a sensitive period in the barn owl. J Neurosci 4:1001-1011

KnUdsen EI, KNudsen PF, Esterly SD (1984b) A critical period for the recovery of sound localization accuracy following monaural occlusion in the barn owl. J Neurosci 4:1012-1020

KOKA K, READ HL, TOLLin DJ (2008) The acoustical cues to sound location in the rat: measurements of directional transfer functions. J Acoust Soc Am 123:4297-4309

KuHn GF (1987) In: Yost WA, Gourevitch G (eds) in directional hearing. Springer-Verlag, New York

Maki K, FurukaWa S (2005) Acoustical cues for sound localization by the Mongolian gerbil, Meriones unguiculatus. J Acoust Soc Am 118:872-886

Margolis RH, Schachern PL, Hunter Ll, Sutherland C (1995) Multifrequency tympanometry in chinchillas. Audiology 34:232247

Middlebrooks JC, Green DM (1990) Directional dependence of interaural envelope delays. J Acoust Soc Am 87:2149-2162

Middlebrooks JC, Pettigrew JD (1981) Functional classes of neurons in primary auditory cortex of the cat distinguished by sensitivity to sound location. J Neurosci 1:107-120

MilLer JD (1970) Audibility curve of the chinchilla. J Acoust Soc Am 48:513-523
Moore DR, Irvine DR (1979) A developmental study of the sound pressure transformation by the head of the cat. Acta Otolaryngol $87: 434-440$

Moore DR, King AJ (2004) Plasticity of binaural systems. SpringerVerlag, New York

Murphy WJ, DAvIS RR (1998) The role of the chinchilla pinna and ear canal in electrophysiological measures of hearing thresholds. J Acoust Soc Am 103:1951-1956

Musicant AD, Chan JC, Hind JE (1990) Direction-dependent spectral properties of cat external ear: new data and crossspecies comparisons. J Acoust Soc Am 87:757-781

Niemiec AJ, Yost WA, Shofner WP (1992) Behavioral measures of frequency selectivity in the chinchilla. J Acoust Soc Am 92:26362649

Nuding SC, Chen GD, Sinex DG (1999) Monaural response properties of single neurons in the chinchilla inferior colliculus. Hear Res 131:89-106

ObRIST MK, Wenstrup JJ (1998) Hearing and hunting in red bats (Lasiurus borealis, Vespertilionidae): audiogram and ear properties. J Exp Biol 201:143-154

Phillips DP, Calford MB, Pettigrew JD, Aitkin LM, Semple MN (1982) Directionality of sound pressure transformation at the cat's pinna. Hear Res 8:13-28

Rice JJ, May BJ, Spirou GA, Young ED (1992) Pinna-based spectral cues for sound localization in cat. Hear Res 58:132-152

Rife DD, VANDERKooy J (1989) Transfer-function measurement with maximum-length sequences. J Audio Eng Soc 37:419-444

Rотн GL, KochHaR RK, HiNd JE (1980) Interaural time differences: implications regarding the neurophysiology of sound localization. J Acoust Soc Am 68:1643-1651

SchnupP JW, Booth J, KIng AJ (2003) Modeling individual differences in ferret external ear transfer functions. J Acoust Soc Am 113:2021-2030

Shofner WP, Yost WA, Whitmer WM (2007) Pitch perception in chinchillas (Chinchilla laniger): stimulus generalization using rippled noise. J Comp Psychol 121:428-439

SLeE SJ, Young ED (2010) Sound localization cues in the marmoset monkey. Hear Res 260:96-108

Spezio ML, Keller CH, Marrocco RT, Takahashi TT (2000) Headrelated transfer functions of the Rhesus monkey. Hear Res 144:73-88

Sterbing SJ, Hartung K, Hoffmann KP (2003) Spatial tuning to virtual sounds in the inferior colliculus of the guinea pig. J Neurophysiol 90:2648-2659

Tilley AR (1993) The measure of man and woman. Henry Dreyfuss Associates, New York

TOLlin DJ, KоKa K (2009A) Postnatal development of sound pressure transformations by the head and pinnae of the cat: binaural characteristics. J Acoust Soc Am 126:3125-3136

Tollin DJ, Kока K (2009в) Postnatal development of sound pressure transformations by the head and pinnae of the cat: monaural characteristics. J Acoust Soc Am 125:980-994

Tollin DJ, YIN TC (2003) Spectral cues explain illusory elevation effects with stereo sounds in cats. J Neurophysiol 90:525-530

von Bismark G (1967) THE sound PREsSURE transformation Function FROM FREE FIELD TO EARDRUM OF THE CHINCHILLA. M.S. thesis, MIT, Cambridge, MA

Xu L, Middeebrooks JC (2000) Individual differences in external-ear transfer functions of cats. J Acoust Soc Am 107:1451-1459 